Discrete Comput Geom 33:67-81 (2005)

DOI: $10.1007 / \mathrm{s} 00454-004-1147-\mathrm{x}$

\title{
Equilibrium Placement of Periodic Graphs and Convexity of Plane Tilings
}

\author{
Olaf Delgado-Friedrichs \\ Wilhelm-Schickard-Institut für Informatik, \\ Universität Tübingen, \\ Sand 14, 72076 Tübingen, Germany \\ delgado@informatik.uni-tuebingen.de
}

\begin{abstract}
We study realizations of periodic graphs in Euclidean spaces with each vertex in the center of gravity of its neighbors. As a first application, we show that every planar, 3-connected, 2-periodic graph can be drawn into the plane with convex faces such that the drawing realizes every combinatorial automorphism of the graph as an isometric symmetry. This extends results by Thomassen and by Mani-Levitska, Guigas, and Klee.
\end{abstract}

\section{Introduction}

Graph embeddings into the ordinary plane have been studied in some detail. Tutte [Tu1], [Tu2] gave criteria for the existence of a convex embedding with a prescribed outer polygon. In particular, every finite 3-connected planar graph has a convex representation. This follows already from Steinitz' theorem [St] asserting that every such graph is the 1-skeleton of some convex three-dimensional polyhedron.

Tutte's proof, apart from being slightly more general, uses a particularly elegant construction in which every inner vertex is placed at the center of gravity of its neighbors. It can be shown that for every placement of the outer vertices, the resulting system of linear equations has a unique solution. Consequently, every symmetry of the outer polygon which is compatible with some automorphism of the graph induces a symmetry of the whole embedding.

Tutte's construction can be interpreted as the equilibrium state of a dynamical system where pairs of adjacent vertices are attracted to each other by forces proportional to the respective edge lengths. Such equilibrium placements have, in turn, been used to prove Steinitz' theorem $[\mathrm{RG}]$.

In [Th1] Thomassen presents generalizations of many standard planarity results to infinite graphs. In particular, he shows that for every 3-connected graph which has a 
planar representation without vertex accumulation points, this representation is essentially unique and can be chosen to be convex. Mani-Levitska et al. [MLGK] provide a related result, showing that every planar, 3-connected, 2-periodic map has a convex, 2periodic realization. Thomassen later presents a simpler proof [Th2], using his own result on criteria for admissible outer polygons in convex representations of 2-connected, finite graphs (also from [Th1]), an extension of Tutte's theorem. Thomassen's technique can be generalized to obtain convex realizations which exhibit more than just the translational symmetries of a given periodic graph [De]. Yet there seems to be no easy way to extend it to the case where some facial cycles are left fixed by automorphisms of the graph.

By generalizing Tutte's technique of barycentric placement, we show here that every planar, 3-connected, 2-periodic graph has an embedding with convex faces such that every automorphism of the graph is induced by an isometric symmetry of the embedding. As a corollary, we establish that every planar, cellular map with a 2-periodic, 2-connected underlying graph has a realization with star-shaped faces such that every map automorphism is induced by an isometric symmetry of that realization.

\section{Periodic Graphs}

Some terminology is given below. Otherwise, we essentially follow Diestel [Di].

We consider simple undirected graphs with positive, real edge weights. For our purposes, a graph is a triplet $(V, E, \omega)$, where $V$ is a non-empty countable set of vertices, $E$ is a set of two-element subsets of $V$, called edges, and $\omega: E \rightarrow \mathbb{R}$ is a function, called a stress, with $\omega(e)>0$ for all $e \in E$. We denote the stress of a graph $G$ by $\omega^{G}$. The $\omega$-image of an edge $v w$, also called the stress on $v w$, will be written $\omega_{v w}$.

For $v \in V$, the set of vertices adjacent to $v$ is called the neighborhood of $v$ in $G$ and denoted $N(v)$. The valency of a vertex is the size of its neighborhood. A graph is locally finite if all its vertices have finite valency. In the following, all graphs are assumed to be locally finite.

If $U \subseteq V$ is a set of vertices, the induced subgraph with vertex set $U$ is denoted $G[U]$. We write $G-U:=G[V \backslash U]$, and, for $H \subseteq G$, we write $G[H]:=G[V(H)]$ and $G-H:=G-V(H)$. When specifying subgraphs in the rest of this paper, we take it that stresses are always inherited from the containing graph.

A path is a finite graph $P$ of the form

$$
V(P)=\left\{v_{0}, \ldots, v_{k}\right\}, \quad E(P)=\left\{v_{0} v_{1}, v_{1} v_{2}, \ldots, v_{k-1} v_{k}\right\},
$$

with the $v_{i}$ pairwise different. We say that $P$ is a path from $v_{0}$ to $v_{k}$ or between $v_{0}$ and $v_{k}$ and that $v_{0}, v_{k}$ are linked by and the ends of $P$.

If $H$ is a subgraph of $G$ and $A$ is a subgraph of $G-H$, then a vertex of attachment of $A$ in $H$ is a vertex $v \in H$ with $N(v) \cap V(A) \neq \emptyset$. An $H$-component of $G$ is either an edge $e \in E(G[H]) \backslash E(H)$ (or, more precisely, the graph $G[\{v, w\}]$, where $e=v w$ ) or the graph induced by some component of $G-H$ together with all its vertices of attachment.

A cycle is a connected graph of size at least 3 , all vertices of which have valency 2 . Where not stated otherwise, cycles are assumed to be finite. A connected graph with no finite cycles is a tree. Every finite tree contains vertices of valency 1 , its leafs. 
A (vertex) traversal of a graph is a finite or infinite alternating sequence

$$
v_{0} e_{1} v_{1} e_{2} v_{2} \ldots
$$

of vertices and edges where $\left\{v_{0}, v_{1}, \ldots\right\}=V(G), v_{i} \neq v_{j}$ if $i \neq j$ and $e_{i}=$ $v_{k_{i}} v_{i}$ for certain $k_{i}<i$. Every connected graph with countably many vertices has a traversal.

Graph isomorphisms in our setting are understood to respect stresses. An automorphism, as usual, is a self-isomorphism of a graph. The group of all automorphisms of a graph $G$ is denoted $\operatorname{Aut}(\mathrm{G})$. For $\varphi \in \operatorname{Aut}(\mathrm{G}), \Phi \subseteq \operatorname{Aut}(\mathrm{G})$ and $H \subseteq G$, we write $\varphi H:=(\varphi(V(H)), \varphi(E(H)))$ and

$$
\Phi H:=\bigcup_{\varphi \in \Phi} \varphi H:=\left(\bigcup_{\varphi \in \Phi} \varphi(V(H)), \bigcup_{\varphi \in \Phi} \varphi(E(H))\right) .
$$

We define a periodic graph as a pair $(G, T)$, where $G$ is an infinite graph and $T$ is a free Abelian subgroup of $\operatorname{Aut}(\mathrm{G})$ which acts on $G$ freely and such that its set $V / T:=\{T v \mid v \in V\}$ of vertex orbits is finite. The group $T$ is called a translation group for $G$ and its rank is called the dimension of $(G, T)$. The pair $(G, T)$ is also called a periodic structure on $G$. A periodic graph of dimension $d$ is called $d$-periodic. The translation group $T$ and the periodic graph $(G, T)$ are both called maximal if no periodic structure $\left(G, T^{\prime}\right)$ exists with $T \lesseqgtr T^{\prime}$. Note that a graph can have more than one, in fact even uncountably many, maximal periodic structures.

An isomorphism between periodic graphs $(G, T)$ and $\left(G^{\prime}, T^{\prime}\right)$, also called a periodic isomorphism and denoted $\varphi:(G, T) \rightarrow\left(G^{\prime}, T^{\prime}\right)$, is an isomorphism $\varphi: G \rightarrow G^{\prime}$ satisfy$\operatorname{ing} \varphi T \varphi^{-1}=T^{\prime}$. A periodic isomorphism $\varphi$ is uniquely determined up to translations by its action on the vertex and edge orbits. This can be seen easily by defining $\varphi$ inductively on a traversal of $G$. As usual, a periodic automorphism is a periodic isomorphism from a periodic graph to itself and the group of all automorphisms of $(G, T)$ is denoted by $\operatorname{Aut}(G, T)$.

We note the following fact on periodic graphs for later reference:

Lemma 1. Let $(G, T)$ be a connected, d-periodic graph, $d \geq 2$, with a finite separating set $U$. Then of the components of $G-U$, exactly one is infinite.

Proof. Let $(G, T), G=(V, E)$ be a counterexample with the minimal number $n$ of vertex orbits. Assume $n>1$. Because $G$ is connected, some edge $e=u v \in E$ must have $u$ and $v$ in different orbits. We consider the graph $G^{\prime}=\left(V^{\prime}, E^{\prime}\right)$ obtained from $G$ by contracting all the edges in the $T$-orbit of $e$. Assume $V^{\prime}:=V \backslash T v$. Then, by construction, the restriction of every $\tau \in T$ to $V^{\prime}$ is an automorphism of $G^{\prime}$. We write $\tau^{\prime}:=\left.\tau\right|_{V^{\prime}}$ and $T^{\prime}:=\left\{\tau^{\prime} \mid \tau \in T\right\}$. There are exactly $n-1$ orbits of $T^{\prime}$ on $V^{\prime}$. If $P$ is a path in $G$ between vertices $a, b \in V^{\prime}$, then there is a similar path in $G^{\prime}$ obtained by replacing every occurrence of $\tau v$ for some $\tau \in T$ by $\tau u$. Consequently, $G^{\prime}$ is connected.

Conversely, if $P^{\prime}$ is a path between $a$ and $b$ in $G^{\prime}$, it is easy to see that there is such a path in $G$ as well. Thus, if $U$ separates $G$, then either $U^{\prime}:=U \cap V^{\prime}$ separates $G^{\prime}$, 
or every component of $G-U$ except one contains only vertices in $T v$. However, if $C$ is an infinite component with, say $V(C)=S v$ for some $S \subseteq T$, then $U$ must contain the infinite vertex set $S u$, a contradiction. Furthermore, if $C$ is an infinite component of $G-U$ with $X:=V(C) \backslash T v$ finite, we can replace $U$ by $U \cup X$ and arrive at the same contradiction. Consequently, for every infinite component of $G-U$ there is an infinite component of $G^{\prime}-U^{\prime}$ and $G^{\prime}$ is a "smaller" counterexample.

For $n=1$, because $G$ is connected, it must contain a subgraph $H$ isomorphic to the $d$-dimensional lattice. Obviously, the lemma holds for $H$. If $H$ does not span $G$, some collection of finitely many translates of $H$ does. Any shortest path between two such translates, say $H$ and $\tau H$, has infinitely many translates which still connect $H$ and $\tau H$. Thus, no pair of translates of $H$ can be separated by a finite set of vertices.

We find it convenient to distinguish between point vectors and distance vectors (which may also be interpreted as translation vectors), where, as usual, the affine space of point vectors is denoted $\mathbb{E}^{d}$ while the linear space of distance vectors is written $\mathbb{R}^{d}$. Thus, the difference of two point vectors is a distance vector and so on. In particular, an affine map on $\mathbb{E}^{d}$ induces a linear map on $\mathbb{R}^{d}$. The group of all affine maps on $\mathbb{E}^{d}$ is denoted $\operatorname{aff}\left(\mathbb{E}^{\mathrm{d}}\right)$.

A placement of a graph $G$ is a function $p: V(G) \rightarrow \mathbb{E}^{d}$ for some $d \in \mathbb{N}$. For an edge $e=v w \in E(G)$, we write $p(v, w)=p(w)-p(v)$. Please note that, unlike before, this notation depends on the order of $v$ and $w$, thus in particular $p(w, v)=-p(v, w)$ and we cannot just write $p(e)$.

A graph automorphism $\varphi$ and a homeomorphism $\alpha: \mathbb{E}^{d} \rightarrow \mathbb{E}^{d}$ are associated with respect to a placement $p$ if $p \circ \varphi=\alpha \circ p$.

If $(G, T)$ is a $d$-periodic graph, a placement of $G$ is called periodic with respect to $T$ if every $\tau \in T$ is associated to some affine translation $\tau^{*}=\tau_{p}^{*} \in \mathbb{R}^{d}$ and the set $T^{*}:=\left\{\tau^{*} \mid \tau \in T\right\}$ spans $\mathbb{R}^{d}$ in the sense that the set of corresponding translation vectors contains a basis for $\mathbb{R}^{d}$. Clearly, then, $\tau^{*}$ must be unique for any given $\tau$ and the map $\tau \mapsto \tau^{*}$ must be a group isomorphism.

Lemma 2. If $(G, T)$ and $\left(G^{\prime}, T^{\prime}\right)$ are d-periodic graphs, $\varphi:(G, T) \rightarrow\left(G^{\prime}, T^{\prime}\right)$ is a periodic isomorphism and $p$ is a periodic placement of $\left(G^{\prime}, T^{\prime}\right)$, then $q:=p \circ \varphi$ is a periodic placement of $(G, T)$.

Proof. Consider some arbitrary $\tau \in T$ and let $\tau^{\varphi}:=\varphi \tau \varphi^{-1}$. By assumption, $\tau^{\varphi} \in T^{\prime}$, so $\left(\tau^{\varphi}\right)_{p}^{*}$ is defined. It follows that

$$
q(\tau v)=p(\varphi \tau v)=p\left(\tau^{\varphi} \varphi v\right)=\left(\tau^{\varphi}\right)_{p}^{*}(p(\varphi v))=\left(\tau^{\varphi}\right)_{p}^{*}(q(v))
$$

holds for every $v \in V(G)$, thus $\tau$ is associated to $\left(\tau^{\varphi}\right)_{p}^{*}$ with respect to $q$ and we may write $\tau_{q}^{*}:=\left(\tau^{\varphi}\right)_{p}^{*}$. Because the map $\tau \mapsto \tau^{\varphi}$ is an isomorphism from $T$ to $T^{\prime}$, we must have

$$
T_{q}^{*}=\left\{\left(\tau^{\varphi}\right)_{q}^{*} \mid \tau \in T\right\}=\left\{\tau_{p}^{\prime *} \mid \tau^{\prime} \in T^{\prime}\right\}=T_{p}^{\prime *} .
$$

Consequently, $q$ is periodic. 


\section{Equilibrium Placements}

A placement $p$ is called in equilibrium or an equilibrium placement if for every vertex $v \in V(G)$ we have

$$
\sum_{w \in N(v)} \omega_{v w} \cdot p(v, w)=0
$$

Proposition 3. Let $G$ and $G^{\prime}$ be graphs with equilibrium placements $p$ and $p^{\prime}$. Let $\alpha$ be an affine transformation such that $\alpha \circ p$ is defined and let $\varphi: V(G) \rightarrow V\left(G^{\prime}\right)$ be an isomorphism from $G$ to $G^{\prime}$. Then $\alpha \circ p$ and $p \circ \varphi$ are in equilibrium, as well.

Proof. As noted before, the affine transformation $\alpha$ induces a linear transformation, also denoted $\alpha$, on the set of distance vectors. Thus,

$$
\begin{aligned}
\sum_{w \in N(v)} \omega_{v w} \cdot(\alpha(p(w))-\alpha(p(v))) & =\sum_{w \in N(v)} \omega_{v w} \cdot \alpha(p(v, w)) \\
& =\alpha\left(\sum_{w \in N(v)} \omega_{v w} \cdot p(v, w)\right)=\alpha(0)=0
\end{aligned}
$$

for all $v \in V$.

Regarding the isomorphism $\varphi$, we obtain

$$
\begin{aligned}
\sum_{w \in N(v)} \omega_{v w} \cdot(p(\varphi w)-p(\varphi v)) & =\sum_{w \in N(v)} \omega_{\varphi(v) \varphi(w)}(p(\varphi w)-p(\varphi v)) \\
& =\sum_{w^{\prime} \in N(\varphi v)} \omega_{\varphi(v) w^{\prime}}\left(p\left(w^{\prime}\right)-p(\varphi v)\right)=0
\end{aligned}
$$

for all $v \in V$.

The proof of the following theorem, establishing existence and uniqueness of equilibrium placements, is an adaption of a similar result from [RG].

Theorem 4. Let $(G, T)$ be a connected, d-periodic graph with $T=\left\langle\tau_{1}, \ldots, \tau_{d}\right\rangle$ and let $t_{1}, \ldots, t_{d}$ be a basis of $\mathbb{R}^{d}$. Then there is a periodic equilibrium placement $p$ of $(G, T)$ with $\left(\tau_{i}\right)_{p}^{*}=t_{i}$ for $i=1, \ldots, d$. Moreover, $p$ is unique up to translations.

Proof. Let $G=(V, E, \omega)$. We construct a minimal, connected, finite subgraph $G_{0}=$ $\left(V_{0}, E_{0}\right)$ of $G$ which contains exactly one vertex from each $T$-orbit. This can be done inductively using the connectedness of $G$. Clearly, $E_{0}$ contains at most one edge from each $T$-orbit on $E$.

We may ignore edges of the form $\{v, \tau v\} \in E$ with $\tau \in T$ because $\omega_{v, \tau v}=\omega_{v, \tau^{-1} v}$ and thus, using the notation $v^{\prime}:=\tau^{-1} v$,

$$
\omega_{v, \tau v} p(v, \tau v)+\omega_{v, \tau^{-1} v} p\left(v, \tau^{-1} v\right)=\omega_{v, \tau v}\left(p(v, \tau v)-p\left(v^{\prime}, \tau v^{\prime}\right)\right)=0 .
$$


Let $E^{\prime}:=\{v w \in E \mid T v \neq T w\}$. By construction, $E_{0}$ is a subset of $E^{\prime}$. Extend $G_{0}$ to a connected graph $\left(V_{1}, E_{1}\right)$ which contains exactly one edge from each $T$-orbit on $E^{\prime}$. Let $V_{0}=\left\{v_{0}, \ldots, v_{k}\right\}$ and $V_{1}=\left\{v_{0}, \ldots, v_{m}\right\}$, where $m \geq k>0$. A periodic placement with prescribed translation vectors is completely determined by its values on $V_{0}$.

For $i \in\{0, \ldots, m\}$ define $r(i) \in\{0, \ldots, k\}$ such that $v_{i}=\tau v_{r(i)}$ for some $\tau \in T$. The vertex $v_{r(i)}$ and the automorphism $\tau$ are both uniquely determined by $i$. For an arbitrary vector $z=\left(x_{0,1}, \ldots, x_{0, d}, \ldots, x_{k, 1}, \ldots, x_{k, d}\right)$, define $p=p_{z}$ as the placement induced by $p\left(v_{i}\right)=\left(x_{i, 1}, \ldots, x_{i, d}\right)$ for $i=0, \ldots, k$ and let

$$
W(z):=\frac{1}{2} \sum_{v w \in E_{1}} \omega_{v w} \cdot\|p(v, w)\|^{2} .
$$

Consider a critical point $z$ of $W$. The partial derivative

$$
\frac{d\left\|p_{z}\left(v_{i}\right)-p_{z}\left(v_{j}\right)\right\|^{2}}{d x_{v, \mu}}
$$

is non-zero if and only if either $r(i)=v$ or $r(j)=v$. Write $E_{v}:=\left\{v_{i} w \in E_{1} \mid r(i)=v\right\}$ and $p(w)=\left(w_{1}, \ldots, w_{d}\right)$ for $w \in V$.

Consequently,

$$
\begin{aligned}
\frac{d W}{d x_{v, \mu}} & =\sum_{v_{i} w \in E_{v}} \omega_{v_{i} w} \cdot\left(w_{\mu}-x_{i, \mu}\right) \\
& =\sum_{v_{v} w \in E^{\prime}} \omega_{v_{v} w} \cdot\left(w_{\mu}-x_{v, \mu}\right)=\sum_{w \in N\left(v_{v}\right)} \omega_{v_{v} w} \cdot\left(w_{\mu}-x_{v, \mu}\right) .
\end{aligned}
$$

The second equality follows because the edges in $E^{\prime}$ which are incident to $v_{v}$ are in one-to-one correspondence to and translates of those edges in $E_{1}$ which are incident to some $v \in V_{1}$ in the orbit of $v_{v}$. Moreover, by construction, we have $p(w)-p(v)=$ $p(\tau w)-p(\tau v)$ for $\tau \in T$. The third equality follows from $(*)$.

Thus, there is a one-to-one correspondence between critical points of $W$ and equilibrium placements of $G$ with the prescribed translation vectors $t_{1}, \ldots, t_{d}$. Because $W$ is invariant with respect to translation, we may assume $p\left(v_{0}\right)=0$ and have to show that the restricted function $W_{0}:=\left.W\right|_{x_{0,1}=\ldots=x_{0, d}=0}$ has exactly one critical point.

If $\|z\|=\left\|\left(x_{1,1}, \ldots, x_{1, d}, \ldots, x_{k, 1}, \ldots, x_{k, d}\right)\right\|>a$ for some positive $a \in \mathbb{R}$, then there is at least one $i \in\{1, \ldots, k\}$ and one $j \in\{1, \ldots, d\}$ with $\left|x_{i, j}\right|>a / \sqrt{k d}$. There is a path in $G_{0}$ of length at most $k$ from $v_{0}$ to $v_{i}$. Thus there is at least one edge $v_{l} v_{r}$ in that path for which

$$
\left|x_{l, j}-x_{r, j}\right| \geq \frac{\left|x_{i, j}\right|}{k}>\frac{a}{\sqrt{k^{3} d}}
$$

This implies

Thus, whenever

$$
W_{0}(z)>\frac{\min (\omega) a^{2}}{2 k^{3} d}
$$

$$
\|z\|>\sqrt{\frac{W_{0}(0) \cdot 2 k^{3} d}{\min (\omega)}},
$$

we have $W_{0}(z)>W_{0}(0)$. 
This implies that the quadratic function $W_{0}$ is non-degenerate and thus has a unique critical point, which in this case is a minimum.

In the rest of this section we show that, for an appropriate choice of metric, every automorphism of a graph in equilibrium placement is associated to an isometry. Unless stated otherwise, let $(G, T)$ be a connected, $d$-periodic graph with $G=(V, E, \omega)$ and $T=\left\langle\tau_{1}, \ldots, \tau_{d}\right\rangle$. Let $p$ be a periodic equilibrium placement of $G$. For $i=1, \ldots, d$, write $t_{i}:=\left(\tau_{i}\right)_{p}^{*}$ and set $T^{*}=T_{p}^{*}:=\left\{\tau^{*} \mid \tau \in T\right\}=\left\langle t_{1}, \ldots, t_{d}\right\rangle$. Let $\Gamma:=\operatorname{Aut}(\mathrm{G}, \mathrm{T})$.

Lemma 5. Let $(G, T)$ and $\left(G^{\prime}, T^{\prime}\right)$ be d-periodic graphs with periodic placements $p$ and $p^{\prime}$, respectively, and let $\varphi:(G, T) \rightarrow\left(G^{\prime}, T^{\prime}\right)$ be a periodic isomorphism. Then there is a unique affine map $\varphi^{*}: \mathbb{E}^{d} \rightarrow \mathbb{E}^{d}$ with $p^{\prime} \circ \varphi=\varphi^{*} \circ p$.

Proof. For $\tau \in T$, set $\tau^{\varphi}:=\varphi \tau \varphi^{-1}$ and $\varphi^{*}\left(\tau_{p}^{*}\right):=\left(\tau^{\varphi}\right)_{p^{\prime}}^{*}$. By assumption, the mapping $\tau \mapsto \tau^{\varphi}$ defines a bijection between $T$ and $T^{\prime}$. It follows that $\varphi^{*}$ maps $T_{p}^{*}$ bijectively onto $T^{\prime *}{ }^{\prime}$. Consequently, $\varphi^{*}$ extends to a unique, well-defined, linear isomorphism of $\mathbb{R}^{d}$. By setting $\varphi^{*}\left(p\left(v_{0}\right)\right):=p^{\prime}\left(\varphi v_{0}\right)$, we obtain a unique, non-singular affine map. Because for every $\tau \in T$, we have

$$
\begin{aligned}
p^{\prime}\left(\varphi \tau v_{0}\right) & =p^{\prime}\left(\tau^{\varphi} \varphi v_{0}\right)=p^{\prime}\left(\varphi v_{0}\right)+\left(\tau^{\varphi}\right)_{p^{\prime}}^{*} \\
& =\varphi^{*}\left(p\left(v_{0}\right)\right)+\varphi^{*}\left(\tau_{p}^{*}\right)=\varphi^{*}\left(p\left(v_{0}\right)+\tau_{p}^{*}\right)=\varphi^{*}\left(p\left(\tau v_{0}\right)\right),
\end{aligned}
$$

the periodic equilibrium placements $p^{\prime} \circ \varphi$ and $\varphi^{*} \circ p$ agree on $T v_{0}$, so by Theorem 4 , they must be identical.

Lemma 6. There is unique group homomorphism

$$
\begin{aligned}
*: \Gamma & \rightarrow \operatorname{aff}\left(\mathbb{E}^{\mathrm{d}}\right) \\
\gamma & \mapsto \gamma^{*}
\end{aligned}
$$

such that $\gamma$ is associated to $\gamma^{*}$ for every $\gamma \in \Gamma$.

Proof. By Lemma 5, the map * exists and is uniquely determined. It remains to show that it is a group homomorphism: by definition, ${ }^{*}$ must map the identity in $\Gamma$ to the identity in aff $\left(\mathbb{E}^{\mathrm{d}}\right)$. For arbitrary $\beta, \gamma \in \Gamma$ and arbitrary $v \in V$, we have

$$
(\beta \gamma)^{*}(p(v))=p(\beta \gamma v)=\beta^{*}(p(\gamma v))=\beta^{*}\left(\gamma^{*}(p(v))\right)=\left(\beta^{*} \gamma^{*}\right) p(v) .
$$

We note some special cases which indicate that, in general, ${ }^{*}$ is not injective.

Corollary 7. If $\gamma$ is an automorphism of $(G, T)$ with $\gamma \tau v_{0}=\tau v_{0}$ for some $v_{0} \in V$ and all $\tau \in T$, then $p(\gamma v)=p(v)$ for all $v \in V$.

Proof. In this case, $\gamma^{*}$ must fix every point of the lattice $p\left(T v_{0}\right)$, so it must be the identity. 


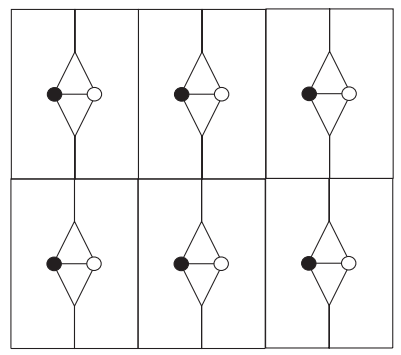

Fig. 1. In an equilibrium placement, vertices marked $\bullet$ and their neighbors marked $\circ$ must receive identical coordinates.

Example 8. Let $G$ be the 2-periodic graph depicted in Fig. 1 and let $\gamma$ be the automorphism which exchanges every vertex marked $\bullet$ with its neighbor marked $\circ$ and leaves the unmarked vertices unchanged.

Corollary 9. If $\gamma$ is an automorphism of $(G, T)$ which commutes with every $\tau \in T$, then $\gamma^{*}$ is a translation. If, moreover, $\gamma$ has finite order, then $\gamma^{*}$ is the identity.

Proof. Because of $\gamma \tau=\tau \gamma$, we have

$$
\gamma^{*}\left(\tau_{p}^{*}(p(v))\right)=p(\gamma \tau v)=p(\tau \gamma v)=\tau_{p}^{*}\left(\gamma^{*}(p(v))\right)
$$

for every $\tau$, so $\gamma^{*}$ is a translation. The second claim follows immediately.

Example 10. The 2-periodic graph depicted in Fig. 2 can be interpreted as two square lattices stacked on top of each other and connected by vertical bars (here represented as diagonals). The automorphism which flips the two lattices fulfills the condition of the corollary, so both lattices will be represented by identical points.

Theorem 11. Let $(G, T), G=(V, E, \omega)$ be a connected, d-periodic graph and let $\Gamma \leq \operatorname{Aut}(\mathrm{G}, \mathrm{T})$ be a group of automorphisms. Then there is a periodic equilibrium placement $p: V \rightarrow \mathbb{E}^{d}$ such that for every $\gamma \in \Gamma$ an isometry $\gamma^{*}: \mathbb{E}^{d} \rightarrow \mathbb{E}^{d}$ associated

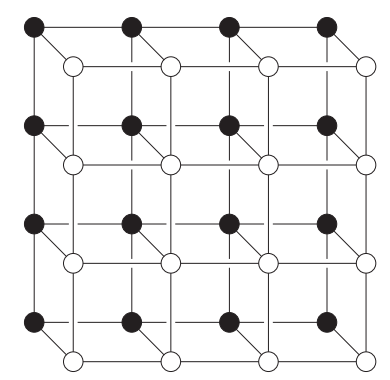

Fig. 2. Another graph in which vertices marked $\bullet$ and their neighbors marked $\circ$ receive identical coordinates. 
to $\gamma$ with respect to $p$ exists. Moreover, $p$ is unique up to affine conjugation and the map $\gamma \rightarrow \gamma^{*}$ is a group homomorphism.

Proof. The existence and uniqueness up to affine conjugation of a periodic equilibrium placement $p$ is established by Theorem 4. A unique group homomorphism ${ }^{*}: \Gamma \rightarrow$ aff $\left(\mathbb{E}^{\mathrm{d}}\right)$ such that for every $\gamma \in \Gamma$, the map $\gamma^{*}$ is associated to $\gamma$, exists by Lemma 6 . It remains to show that there is indeed a choice of $T^{*}$ which turns all these affine maps into isometries.

Consider $P:=\rho\left(\left\{\gamma^{*} \mid \gamma \in \Gamma\right\}\right)$, where $\rho$ is the group homomorphism that maps an affine transformation to its linear constituent. As noted above, every automorphism of $(G, T)$ is, up to translations, uniquely determined by its action on the finitely many vertex and edge orbits. Consequently, $P$ is finite and by the usual trick, we find a metric on $\mathbb{E}^{d}$ with respect to which $P$ acts isometrically, namely by defining a new scalar product $s$ as $s(v, w):=\sum_{\alpha \in P}\langle\alpha v, \alpha w\rangle$.

An orthonormal basis with respect to $s$ can then be found by Gram-Schmidt orthonormalization. Thus, after fixing an origin, $p$ can be expressed, if required, in terms of the usual basis and metric for $\mathbb{R}^{d}$.

Apart from the application given below, Theorem 11 is also the key to identifying ideal symmetries in higher-dimensional periodic graphs, which, as the method of equilibrium placement itself, has important applications in crystallography (see [DFO]).

\section{Convex Embeddings of Planar Graphs}

A planar graph is one that can be drawn into the plane without intersections. More generally, we define a drawing of a graph as a collection of points and arcs where each vertex is represented by a single point and each edge by a single arc connecting the points which represent its ends. A drawing is an embedding if the vertex placement is injective and no edge arc crosses any other edge arc or passes through any vertex position. To avoid technical complications, we assume all drawings to be piecewise linear.

A straight line drawing is one in which all edges are represented by straight lines. Clearly, every placement $p: V(G) \rightarrow \mathbb{E}^{d}$ induces a unique straight line drawing of $G$ in $\mathbb{E}^{d}$.

A graph is planar if it has an embedding into the Euclidean plane. The faces of that embedding are then the pathwise connected components of its complement.

The aim of this section is to show that the straight line drawing induced by an equilibrium placement of a 2-periodic, 3-connected planar graph is always an embedding. We start by showing that planar 2-periodic graphs have "nice" embeddings in the plane.

It is well known that for finite 2-connected graphs, each face of a plane embedding is bounded by the representation of some cycle, which is then called a facial cycle. If $G$ is 3-connected, then a theorem by Whitney [Wh] states that the set of facial cycles does not depend on the particular embedding. For infinite planar graphs, the situation can be more complicated. We will, however, establish a close analogue to Whitney's theorem for planar, 2-periodic graphs. 
Following [Th1], we define a vertex accumulation point (abbreviated VAP) in a graph drawing as some point $p$ such that in every open $\varepsilon$-ball around $p$ there are infinitely many realizations of vertices. An edge accumulation point or EAP is defined analogously. The key result for us is the following easy corollary to Theorem 7.4 of [Th1].

Lemma 12. Let $G$ be a 3-connected, infinite graph which has a VAP-free embedding into the plane. Then the set of facial cycles of any such embedding is unique. Moreover, the embedding can be chosen to be EAP-free as well.

We call a 2-periodic graph $(G, T)$ a grid if it can be derived from either the underlying graph of the regular square or the regular honeycomb tiling, each equipped with its maximal translation group, by periodic subdivision.

Lemma 13. The set of facial cycles in any planar, VAP-free embedding of a grid is unique.

Proof. This follows immediately from Lemma 12.

Lemma 14. Let $(G, T)$ be a connected, 2-periodic graph. Then there is a grid $\left(H, T^{\prime}\right)$ with $H$ a subgraph of $G$ and $T^{\prime} \leq T$.

Proof. Let $r$ be a non-trivial path of minimal length between vertices $v$ and $\tau v$, where $\tau \in T$. By construction, $\tau$ cannot have an interior vertex in common with any of its translates. Consequently, the graph $R:=\langle\tau\rangle r$ is an infinite cycle.

Now let $s$ be a non-trivial path of minimal length between $R$ and $\sigma R$ for some $\sigma \in T$, where $\tau$ and $\sigma$ are linearly independent. Again, $s$ cannot intersect any of its translates.

However, then, with $T^{\prime}:=\langle\sigma, \tau\rangle$, the graph $\left(T^{\prime} r \cup T^{\prime} s, T^{\prime}\right)$ is a grid.

Theorem 15. Every planar, 3-connected, 2-periodic graph $(G, T)$ has a planar drawing which is VAP-and EAP-free. The set of facial cycles of any such drawing is unique. Moreover, it can be chosen to be periodic with respect to some finite index subgroup $T^{\prime}$ of $T$.

Proof. Let $(G, T)$ be a planar, 3-connected, 2-periodic graph and let $\left(H, T^{\prime}\right)$ be a grid with $H \subseteq G$ and $T^{\prime} \leq T$. Let $A$ be any $H$-component in $G$. Then, obviously, $A$ must be a $C$-component for some facial cycle $C$ of $H$. By Lemma $1, A$ must be finite, for $G$ already has an infinite $C$-component containing $H$.

Moreover, $C \cup A$ must have a planar drawing with $C$ being one of its facial cycles and we may draw $A$ into the face $f$ of the drawing which is bounded by $C$. Now consider two $C$-components $A$ and $A^{\prime}$ that cannot be drawn into $f$ simultaneously. This can only happen if one of these, say $A^{\prime}$, is also a $C^{\prime}$-component for $C^{\prime}$ some adjacent facial cycle in $H$. Consequently, $A^{\prime}$ must have all its vertices of attachment on the common path which forms the intersection of $C$ and $C^{\prime}$. However, because $G$ is 3-connected, it is easy to adjust $H$ in such a way that no such component exists. 
We may thus take the standard drawing of $H$ and extend it to a VAP- and EAPfree drawing of $G$ in a $T^{\prime}$-periodic way. The uniqueness of facial cycles follows from Lemma 12 again.

We proceed to show the convexity result itself. Our proof is an adaption of RichterGebert's $[R G]$ strategy for the finite case. It turns out useful to study a slightly more general property: the relative interior of a set of points $\left\{s_{1}, \ldots, s_{k}\right\}$ is the set

$$
\left\{\sum_{i=1}^{k} \lambda_{i} s_{i} \mid \sum_{i=1}^{k} \lambda_{i}=1 ; \lambda_{i}>0 \text { for } i=1, \ldots, k\right\} .
$$

A well-known result from the theory of convex sets implies that the relative interior thus defined is precisely the interior of the convex hull of $\left\{s_{1}, \ldots, s_{k}\right\}$ with respect to its affine hull.

A placement $p$ is called good if, for every $v \in V, p(v)$ is in the relative interior of the set $p(N(v))$. A placement is non-degenerate if, for every $v \in V, p(N(v))$ affinely spans $\mathbb{E}^{2}$.

We show a special case first:

Lemma 16. Let $(G, T)$ be a planar, 2-periodic, 3-connected graph, all facial cycles of which are triangles. Let $p: V \rightarrow \mathbb{E}^{2}$ be a non-degenerate, good, 2-periodic placement which maps the vertices of each triangle to affinely independent points. Then the straight line drawing induced by $p$ is an embedding.

Proof. Let $F$ be the set of facial cycles of $G$. To each face, assign an orientation, i.e., a cyclic order on its vertices, in such a way that the orientations on an edge induced by its two adjacent faces are always opposite. This is possible because the plane is oriented and we already know that $G$ has a planar embedding. If $u, v, w$ are the vertices of some face according to that order and $u^{\prime}, v^{\prime}, w^{\prime}$ their respective $p$-images, we denote by $a(u, v, w)$ the signed angle $\varangle u^{\prime} v^{\prime} w^{\prime}$ at $v^{\prime}$, which is understood to be a number between $-\pi$ and $\pi$. The angles $a(u, v, w), a(v, w, u)$, and $a(w, u, v)$ are either all positive or all negative and their sum is either $\pi$ or $-\pi$.

The orientation of triangles also induces a cyclic order on the neighborhood of each vertex. For an arbitrary vertex $v$, let the ordered sequence of neighbors be $w_{0}, \ldots, w_{k-1}$. Define $a_{i}:=a\left(w_{i}, v, w_{i+1}\right)$ for $i=0, \ldots, k-1$, with indices to be read modulo $k$. Set

$$
\Psi(v):=\sum_{i=0}^{k-1} a_{i} \quad \text { and } \quad \Phi(v):=\sum_{i=0}^{k-1}\left|a_{i}\right| .
$$

Moreover, let

$$
\Psi^{+}(v):=\sum_{a_{i}>0} a_{i} \quad \text { and } \quad \Psi^{-}(v):=\sum_{a_{i}<0} a_{i} .
$$

Then $\Psi(v)=\Psi^{+}(v)+\Psi^{-}(v)$ and $\Phi(v)=\Psi^{+}(v)-\Psi^{-}(v)$. In particular, we have $\Phi(v) \geq|\Psi(v)|=2 n \pi$ for some $n \in \mathbb{N}$. The case $n=0$ is special and implies $\Psi^{+}(v)=-\Psi^{-}(v)$ and $\Phi(v)=2 \Psi^{+}(v)$. If $\Psi^{+}(v) \leq \pi$, there must be a straight 
line $g$ through $p(v)$ such that $p(N(v))$ is contained in one of the closed half-spaces determined by $g$. However, this contradicts the assumption that $p$ is a non-degenerate good placement. Consequently, $\Phi(v) \geq 2 \pi$ and, in case of equality, all the $a_{i}$ must have the same sign.

By Theorem 15, it follows that the translates of facial cycles are, again, facial cycles. We conclude further that, without loss of generality, there exists a grid $(H, T)$ with $H \subseteq G$. Each face $f$ of the standard drawing of $H$ contains exactly one face for each $T$-orbit on the set of facial cycles of $G$. Identifying boundary points and arcs of $f$ which represent vertices or edges, respectively, in common $T$-orbits yields a cellular subdivision (or tiling) of the torus with $n=\#(F / T)$ faces, $m=\#(E / T)$ edges, and $r=\#(V / T)$ vertices.

The Euler characteristic of the torus is 0 , so we have $n-m+r=0$. On the other hand, the faces are still triangles, so $3 n=2 m$ and consequently $n=2 r$. In each triangle, the sum of absolute values of angles is $\pi$, so the sum of all these angles is $2 r \pi$. On the other hand, the absolute values of angles around each vertex sum up to some $\Phi(v) \geq 2 \pi$ for each of the $r$ vertices. Consequently, the sum of angles around any given vertex must be either $2 \pi$ or $-2 \pi$ and all these angles must have the same sign. Because the angles in any given triangle have the same sign, we may assume without loss of generality that all angles are positive.

Now consider a continuous map $\varphi: \mathbb{E}^{2} \rightarrow \mathbb{E}^{2}$ which maps each face of a given embedding of $G$ homeomorphically onto the corresponding triangle determined by $p$. Obviously, $\varphi$ can be defined separately for each face and adjusted in such a way that it is well-defined on the arcs and points as well. Because the angles of these triangles are all positive and sum up to $2 \pi$ at every vertex, $\varphi$ is a covering. Since $\mathbb{E}^{2}$ is simply connected, it must then be a homeomorphism, which proves the lemma.

Lemma 17. Let $(G, T)$ be a planar, 2-periodic, 3-connected graph. Then every good, periodic placement of $(G, T)$ is non-degenerate.

Proof. Assume $G=(V, E, \omega)$ and let $p$ be a good 2-periodic placement of $G$. Suppose that $v \in V$ is a degenerate vertex, i.e., all neighbors of $v$ are placed on a common straight line $L$. Without loss of generality, we may assume $L=\{(x, 0) \mid x \in \mathbb{R}\}$. We denote the second coordinate of $p(w)$ by $h(w)$ and, by a slight abuse of language, call it the height of $w$. Because $p$ is good, $p(v) \in L$, or, equivalently, $h(v)=0$.

The placement $p$ is periodic, so there must be a vertex $u$ with $h(u) \neq 0$. Because $G$ is 3-connected, by Menger's theorem there are at least three paths in $G$ from $v$ to $u$ with pairwise disjoint interiors. Let $a_{0}, b_{0}$, and $c_{0}$ be the respective first vertices on these paths which have neighbors not placed on $L$. Obviously, $p\left(a_{0}\right), p\left(b_{0}\right)$, and $p\left(c_{0}\right)$ are all still on $L$.

Let $a_{1} \in N\left(a_{0}\right)$ be a vertex not placed on $L$. Without loss of generality, we may assume $h\left(a_{1}\right)>0$. Because $p$ is good and there is a vertex adjacent to $a_{1}$, namely $a_{0}$, with $h\left(a_{0}\right)<h\left(a_{1}\right)$, there must be another one, say $a_{2}$, with $h\left(a_{2}\right)>h\left(a_{1}\right)$. By induction, there is an infinite, strictly ascending walk $\left(a_{i}\right)_{i \in \mathbb{N}_{0}}$ in $G$ starting at $a_{0}$.

Because $V / T$ is finite, there are numbers $m>k \geq 0$ and an automorphism $\rho \in T$ such that $a_{m}=\rho a_{k}$. Because $h\left(\rho a_{k}\right)=h\left(a_{m}\right)>h\left(a_{k}\right)$ and $\rho$ is associated to a translation, we have $h(\rho w)>h(w)$ for all $w \in V$. In particular, $h\left(\rho a_{i}\right)>h\left(a_{i}\right)$ for 
$i=0, \ldots, k-1$ and so the walk $a_{0}, \ldots, a_{m}\left(=\rho a_{k}\right), \rho a_{k-1}, \ldots, \rho a_{0}$ from $a_{0}$ to $\rho a_{0}$ is, except for $a_{0}$, completely above $L$. Moreover, because there is a path, and therefore a walk, placed on $L$ from $v$ to $a_{0}$, the translated walk from $\rho v$ to $\rho a_{0}$ is above $L$, and we can extend the above-mentioned walk from $a_{0}$ to $\rho a_{0}$ to one from $a_{0}$ to $\rho v$, still above $L$. Moreover, for every $\rho^{\prime} \in T$ with $h\left(\rho^{\prime} a_{0}\right)>h\left(a_{0}\right)$ there is even a walk from $\rho^{\prime} v$ to $\rho \rho^{\prime} v=\rho^{\prime} \rho v$ placed completely above $L$.

Applying the same reasoning for $b_{0}$ and $c_{0}$, we find elements $\sigma$ and $\tau$ in $T$ such that $b_{0}$ is connected to $\sigma v$ and $c_{0}$ to $\tau v$ by walks placed above $L$ except at $p\left(b_{0}\right)$ and $p\left(c_{0}\right)$, respectively. For each $\rho^{\prime}$ as before, there are walks from $\rho^{\prime} v$ to $\sigma \rho^{\prime} v$ and $\tau \rho^{\prime} v$, respectively, all above $L$.

Putting things together, we can connect $a_{0}, b_{0}$, and $c_{0}$ to $\rho \sigma \tau v$ by walks which all stay above $L$. In other words, there is a connected subgraph of $G$ containing $a_{0}, b_{0}$, and $c_{0}$ with all other vertices placed above $L$. Let $H_{+}$be such a subgraph which has minimal numbers of vertices and edges. It follows that $H_{+}$is a tree with leafs $a_{0}, b_{0}$, and $c_{0}$ and no other leafs. For the moment, such a tree is called a Y-graph.

An analogous construction yields a second Y-graph $H_{-}$with leafs $a_{0}, b_{0}$, and $c_{0}$ and all other vertices placed below $L$. Finally, there is a Y-graph $H_{0}$ with the same leafs placed completely on $L$, as established earlier. These three Y-graphs together form a subgraph of $G$ which is a subdivision of the complete bipartite graph $K_{3,3}$. This cannot happen if $G$ is planar. It follows that our initial assumption was false and $p$ is non-degenerate.

Corollary 18. Let $(G, T)$ be a planar, 2-periodic,3-connected graph. Then the set of all good, periodic placements inducing the same prescribed association map $*: T \rightarrow \operatorname{aff}\left(\mathbb{E}^{2}\right)$ is open.

Proof. If for some $v \in V$ the set $p(N(v))$ affinely spans $\mathbb{E}^{2}$ and $p(v)$ is in the relative interior of $p(N(v))$, this remains true if any of these points is moved within some small neighborhood. It follows that a small enough perturbation of a good placement is still good.

Theorem 19. Let $(G, T), G=(V, E)$ be a planar, 2-periodic, 3-connected graph. Then every good, periodic placement $p$ of $(G, T)$ induces a straight-line embedding with convex faces.

Proof. First construct a planar triangulation of $G$, i.e., a planar graph $G^{\prime}=\left(V^{\prime}, E^{\prime}\right)$ with $V^{\prime}=V$ such that all faces of $G^{\prime}$ are triangles. This can be done, for example, by choosing a vertex $v$ in every face of $G$ and connecting it with all vertices in the same face except for $v$ itself and its immediate neighbors. Picking these vertices consistently with $T$ ensures that $G^{\prime}$ is periodic. If $p$ is a good placement for $G$, then it is also a good placement for $G^{\prime}$.

Suppose there is some triangle $t$ of $G^{\prime}$ with vertices $a, b$, and $c$ all placed on a common straight line. Because the argument does not depend on a particular choice of $T$, we may assume that these vertices belong to pairwise different $T$-orbits. Replacing, if necessary, $p$ by some slightly perturbed, but still good and periodic placement $p^{\prime}$, we may further 
assume that no two vertices of $t$ are mapped to the same point and that no triangle outside the $T$-orbit of $t$ is degenerate. Let $s:=\left[p^{\prime}(a), p^{\prime}(c)\right]$ and assume $p^{\prime}(b) \in s$.

Because $p^{\prime}$ is good, the vertex $b$ has neighbors on both sides of $s$. By Corollary 18 again, there is a good, periodic placement $p^{\prime \prime}$ with no degenerate triangles and $p^{\prime \prime}(b)$ slightly off $s$ such that for some neighbor $d$ of $b$ the straight-line segments between $\left[p^{\prime \prime}(a), p^{\prime \prime}(c)\right]$ and $\left[p^{\prime \prime}(b), p^{\prime \prime}(d)\right]$ intersect. This is a contradiction to Lemma 16.

Consequently, there are no degenerate triangles for $p$ and, again by Lemma 16, the straight-line placement of $G^{\prime}$ induced by $p$ is an embedding. Because $G$ is just a subgraph of $G^{\prime}, p$ induces a straight-line embedding of $G$. The convexity of the faces then follows immediately from the fact that $p$ is good.

Theorem 20. Let $(G, T)$ be a 2-periodic, 3-connected planar graph. Then $G$ has an embedding into the plane with convex faces such that every automorphism of $G$ is induced by an isometric symmetry of the embedding.

Proof. By Theorem 15, the facial cycles of $G$ do not depend on the embedding. Consequently, every automorphism of $G$ must map facial cycles onto facial cycles and, furthermore, induce a topological automorphism of the tiling defined by the embedding. Because of periodicity, there are only finitely many Aut $(\mathrm{G})$-orbits on the set of faces. By a well-known result from tiling theory, a tiling with this property is topologically equivalent to one in which every topological automorphism is realized as an isometry (see $[\mathrm{DH}]$ and $[\mathrm{Sc}]$ ). Because of the structure of crystallographic groups, this implies that there is a unique maximal translation group for $G$, which is normal in $\operatorname{Aut}(\mathrm{G})$. We may therefore apply Theorem 11, which asserts the existence of an equilibrium placement in which every automorphism of $G$ is associated to an isometry. Finally, Theorem 19 shows that the induced straight-line embedding is convex.

Corollary 21. Let $(G, T)$ be a 2-periodic, 2-connected planar graph. Let $\eta$ be an embedding of $G$ with a $T$-invariant set of facial cycles. Then $G$ has a straight-line embedding into the plane with star-shaped faces such that every automorphism of $G$ which maps facial cycles onto facial cycles is induced by an isometric symmetry of the embedding.

Proof. Construct a triangulation $G^{\prime}$ by introducing a new vertex for each facial cycle and connecting it to every vertex in the cycle by a single edge. Then $G^{\prime}$ is 3-connected and every automorphism of $G$ which respects the facial cycles can be extended uniquely to an automorphism of $G^{\prime}$. Consequently, $G^{\prime}$ has a convex embedding with full symmetry, which implies the desired embedding for $G$.

\section{References}

[De] O. Delgado-Friedrichs. Die automatische Konstruktion periodischer Pflasterungen. Master's thesis, University of Bielefeld, 1990.

[DF0] O. Delgado-Friedrichs and M. O'Keeffe. Identification of and symmetry computation for crystal nets. Acta Crystallogr. Sect. A., 59(4):351-360, 2003. 
[DH] A.W.M. Dress and D.H. Huson. On tilings of the plane. Geom. Dedicata, 24:295-310, 1987.

[Di] R. Diestel. Graph Theory. Second edition. Springer-Verlag, Heidelberg, 2000.

[MLGK] P. Mani-Levitska, B. Guigas, and W.E. Klee. Rectifiable $n$-periodic maps. Geom. Dedicata, 8:127$137,1979$.

[RG] Jürgen Richter-Gebert. Realization Spaces of Polytopes. Springer-Verlag, Berlin, 1996.

[Sc] P. Schmitt. $n$-Homeohedral types of tilings. Geom. Dedicata, 32(3):319-327, 1989.

[St] E. Steinitz. Polyeder und Raumeinteilungen. Enzykl. math. Wiss., 3:1-139, 1922.

[Th1] C. Thomassen. Planarity and duality of finite and infinite graphs. J. Combin. Theory. Ser. B, 29:244-271, 1980.

[Th2] C. Thomassen. Plane representations of graphs. In Progress in Graph Theory (Waterloo, Ont., 1982), pages 43-69. Academic Press, Toronto, ON, 1984.

[Tu1] W.T. Tutte. Convex representations of graphs. Proc. London Math. Soc. (3), 10:304-320, 1960.

[Tu2] W.T. Tutte. How to draw a graph. Proc. London Math. Soc., 13:743-767, 1963.

[Wh] H. Whitney. 2-Isomorphic graphs. Amer. J. Math., 55:245-254, 1933.

Received August 18, 2002, and in revised form June 8, 2004. Online publication October 25, 2004. 